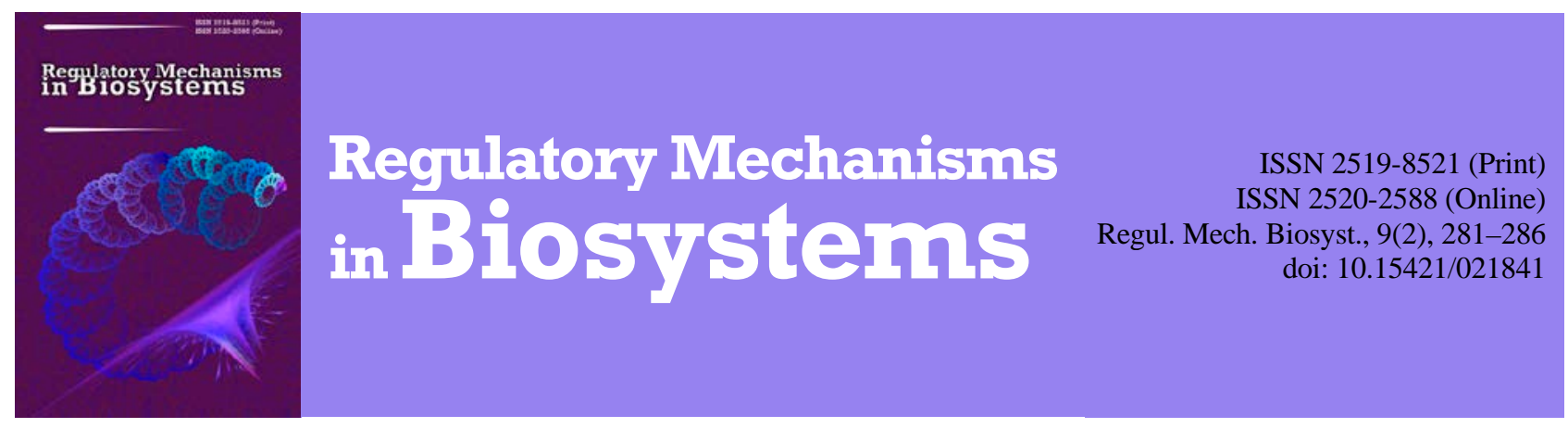

\title{
Monitoring of multiresistant community-associated MRSA strains from patients with pathological processes of different localization
}

\author{
T. V. Sklyar*, K. V. Lavrentieva*, V. G. Gavrilyuk*, N. V. Kurahina*, M. O. Vereshchaha, O. A. Lykholat** \\ *Oles Honchar Dnipro National University, Dnipro, Ukraine \\ **University of Customs and Finance, Dnipro, Ukraine
}

Article info

Received 30.03.2018

Received in revised form 21.04.2018

Accepted 27.04.2018

Oles Honchar Dnipro National University, Gagarin av., 72, Dnipro, 49010, Ukraine. Tel.: +38-050-94-00-098. E-mail: microviro@ukr.net

University of Customs and Finance,

Volodymyr Vernadsky st.,2/4, Dnipro, 49000 Ukraine.

Tel.: +38-056-247-18-82.

\begin{abstract}
Sklyar, T. V., Lavrentieva, K. V., Gavrilyuk, V. G., Kurahina, N. V., Vereshchaha, M. O., \& Lykholat, O. A. (2018). Monitoring of multiresistant community-associated MRSA strains from patients with pathological processes of different localization. Regulatory Mechanisms in Biosystems, 9(2), 281-286. doi:10.15421/021841
\end{abstract}

The therapy of infections, caused by methicillin-resistant Staphylococcus aureus (MRSA) with multiple resistance to antibiotics remains one of the most acute problems all over the world. It is all the more complicated since a priori the MSRA strains are not sensitive to the group of $\beta$-lactam antibiotics and multiresistant isolates are resistant to other groups of antimicrobial preparations, including antibiotics of choice (rifampicin, vancomycin, fusidic acid, co-trimoxazole and linezolid). From the samples of biomaterials of patients with pathological processes of different localization, we isolated 335 strains of bacteria, which were identified as Staphylococcus aureus, 169 (50.4\%) of which were methicillin-resistant variants: $57.5 \%$ cultures were isolated from the nasal discharge; $50.7 \%$ - from faeces at intestinal dysbioses; by $40.0 \%$ - from conjunctival discharge, pharyngeal swab, outer ear swab and sputum; 33.3\% - from urine samples. Antibiotic susceptibility of the isolated cultures was estimated by the disc-diffusion method and the method of serial dilution. The MRSA strains appeared to be most resistant to gentamycin, erythromycin (by 59.5\% of cultures) and ciprofloxacin (53.3\% of isolates), most sensitive - to vancomycin, co-trimoxazole and fusidic acid. The frequency of isolation of the cultures that are resistant to antibiotics did not exceed 4.1\%. Rifampicin suppressed the growth of $75.8 \%$ and linezolid - of $100.0 \%$ of strains. Depending on the kind of biomaterial taken, MRSA strains, isolated from the nasal cavity, outer ear, urine samples, samples of sputum and faeces at intestinal dysbioses proved to be most resistant to the tested antimicrobial preparations. Rifampicin- and vancomycin-resistant strains of methicillin-resistant staphylococci made up $21.3 \%$ of the total number of the detected MRSA. They were most often isolated from the clinical samples taken from the nasal cavity and faeces. When determining minimal inhibitory concentration (MIC) of rifampicin and vancomycin, which are antibiotics of choice for treatment of infections caused by multiresistant MRSA, it was found that for 55.5\% of the MRSA strains isolated from faeces, MIC of rifampicin coincided with the threshold value for this antibiotic and for $44.5 \%$, it exceeded the threshold value by 2 times ( 4 $\mu \mathrm{g} / \mathrm{ml}$ ). $22.2 \%$ of them were characterized by the critical value of susceptibility to vancomycin (MIC $\geq 2 \mu \mathrm{g} / \mathrm{ml}$ ). From rifampicin- and vancomycin-resistant MRSA stains, isolated from the nasal cavity, MIC of rifampicin coincided with the threshold value for this antibiotic for $66.7 \%$ of cultures, and exceeded it at least by 2 times for $33.3 \%$. $11.1 \%$ of them were characterized by the critical level of susceptibility to vancomycin (MIC $\geq 2 \mu \mathrm{g} / \mathrm{ml}$ ) and by $3.7 \%$ of strains exceeded MIC by 2 and 4 times respectively ( 4 and $8 \mu \mathrm{g} / \mathrm{ml}$ ).

Keywords: Staphylococcus aureus; rifampicin; vancomycin; fusidic acid; co-trimoxazole; linezolid

\section{Introduction}

Staphylococcus infections have become widespread lately and they flow in a much more complicated way than 20-30 years ago (DeLeo et al., 2010; Dhanoa et al., 2012; Ehelepola et al., 2018). They are characterized by a considerable variety of localization of the pathogen and clinical manifestation, which complicates their diagnosis (Tong et al., 2015; Hibbitts et O'Leary, 2018).

Staphylococcus aureus is one of the most common microorganisms that causes a wide range of human diseases. It is capable of affecting almost all organs and tissues, demonstrating in this case a whole range of adaptive capabilities. However, the problem is not only the considerable propagation range and high frequency of isolation of $S$. aureus, but also the high level of its antibiotic resistance.

Methicillin-resistant strains of $S$. aureus (MRSA) are the most dangerous in medical practice. In most countries (the USA, Latin America, SAR, India and China), the frequency of their isolation is $25.0 \%$ of the total number of S. aureus strains (Oh et al., 2018; Para et al., 2018). MRSA are characterized by a high level of resistance to antibiotics of different pharmacological groups - macrolides, aminoglycosides, fluoroquinolones, especially to $\beta$-lactam group, including penicillin and cephalosporin of all generations (Gostev et al., 2015; Khan et al., 2018). Resistance of MRSA to $\beta$-lactam antibiotics is caused by the synthesis of penicillin-binding protein (PBP2A) by bacterial cells, which is coded by gene mecA and is characterized by high affinity to $\beta$-lactams (Aguayo-Reyes et al., 2018; Pardos de la Gandara et al., 2018).

Up to the end of the 1990s, MRSA strains were exclusively pathogens of nosocomial infections. In the last decades, the morbidity rate for MRSA-caused nosocomial infections has considerably decreased due to implementation of programs of infection control in hospitals. At the same time, the community-associated strains of methicillin-resistant staphylococci, which maintain constant circulation of variants with multiple resistance to antibiotics, are found increasingly often (García-Garrote et al., 2014; Becker et al., 2018). Community-associated MRSA strains differ from nosocomial ones 
by genotype and phenotype and have a special IV type of Staphylococcus chromosome cassette that is normally resistant to $\beta$-lactam antibiotics but more sensitive to fluoroquinolones and clindamycin. In addition, community-associated MRSA strains differ by high virulence, caused by synthesis of exotoxins, and, primarily, PantonValentine leucocidin (Gomes et al., 2015; Bakthavatchalam et al., 2017).

For a long time, vancomycin remained the preparation of choice for treating infections caused by MRSA strains. But in the middle of the 1990s, first in Japan then in the USA, after that in other countries of the world, reports about more and more frequent isolation of methicillinresistant staphylococci with decreased susceptibility to vancomycin started to appear: heterogeneous vancomycin-intermediate $S$. aureus (hVISA) and vancomycin-intermediate S. aureus (VISA) (Howden, 2014; Gostev et al., 2015; Sirichoat et al., 2016; Tran \& Rybak, 2018). Due to this fact, new preparations for treating infections caused by methicillin-resistant staphylococci were implemented to medical practice: daptomycin, linezolid, ceftaroline, tigecycline. However, the resistance mechanisms of Staphylococcus spp. to these antibiotics have been also described today (Rybak et al., 2014; Miller et al., 2016; Sakoulas et al., 2017). Monitoring of the level of resistance both to traditional preparations and to the new ones is an important element in formation of a strategy of rational antibacterial therapy of infections caused by MRSA.

Based on the relevance of the topic, the aim of the research is the analysis of the level of resistance of MRSA strains, isolated from patients with pathological processes of different localization, to antibiotics of different pharmacological groups, used in clinical practice for treating staphylococcus infections.

\section{Materials and methods}

The object of the research is samples of biomaterial from patients with pathological processes of different localization. The subject is antibiotic resistance of methicillin-resistant stains of $S$. aureus, isolated from the studied biomaterial samples.

Sampling was carried out in accordance with the guidelines on observance of sanitary-epidemiology regime in health care establishments of Ukraine. Samples of urine, faeces at intestinal dysbioses, nasal and conjunctival discharges, pharyngeal swab, outer ear swab and sputum were used as clinical material.

The samples of the clinical material were inoculated on the blood and yolk-salt agars. Inoculated media were controlled thermostatically at $37^{\circ} \mathrm{C}$. After $18-24 \mathrm{~h}$, microscopy of the growing colonies was performed. Only the colonies in which the microscopy revealed grampositive cocci, arranged in the form of clusters of irregular shape, were inoculated on differential-diagnostic media for further identification. For the final identification of the isolated cultures, existence of such physiological and biochemical properties as lecithinase activity, ability to plasmocoagulase synthesis and mannitol fermentation were additionally investigated.

Lecithinase activity was tested on yolk-salt agar. Some strains of S. aureus formed a turbidity area around the colony, i.e. had a positive lecithinase reaction. Further, plasma coagulating activity in the reaction of blood plasma coagulation was determined. If both tests were

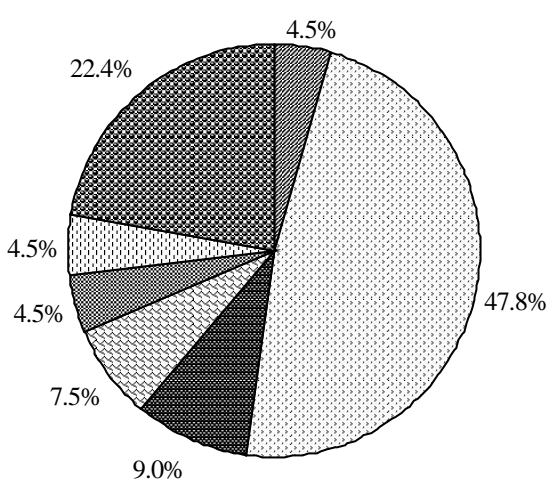

Fig. 1. Isolation of Staphylococcus aureus strains from samples of biomaterial from patients with pathological processes of different localization $(n=335)$ positive, the belonging of the isolated cultures to $S$. aureus was proved. If the cultures had only plasma coagulation or only lecithinase activity, the test on mannitol fermentation was conducted. S. aureus strains, unlike other staphylococci, fermented mannitol. The percenttage of detection of methicillin-resistant strains of $S$. aureus was determined by inoculation of isolated cultures of $S$. aureus on meat infusion agar with methicillin (oxacillin) and cefoxitin.

Subsequently, susceptibility of the isolated strains of methicillinresistant staphylococci to a range of antibiotic drugs of different pharmacological groups was determined using the disc-diffusive method and the method of minimal serial dilution (ISO20776-1:2006). When using the disc-diffusion method, the standard discs with antibiotics were used. The spectrum of antibiotic preparations included: tetracycline, ciprofloxacin, moxifloxacin, erythromycin, clindamycin, cotrimoxazole, vancomycin, rifampicin, gentamycin, linezolid, fusidic acid. Optical density of the bacterial suspension for each of the test cultures, by the Macfarland standard, was brought to 0.5 before inoculation of the medium. The content of the microbial cells was $1,5 \times 10^{8} \mathrm{CFU} / \mathrm{cm}^{3}$.

The referent method for determining susceptibility of the selected pathogens to antibiotics is the method of serial dilution. It also allows determination of the magnitude of minimum inhibitory concentration (MIC) of an antibiotic relative of the tested strains. This is especially important in isolation of multiresistant MRSA from the clinical material. The range of antibiotics for determining MIC in relation to multiresistant MRSA includes rifampicin and vancomycin. These antibiotics are the preparations of choice in treatment of infections, caused by isolates of methicillin-resistant staphylococci with multiple resistance to antibiotics, and the situation of exceeded threshold values to them is estimated as extremely dangerous.

When applying the method of serial dilution, the daily culture of staphylococci was used to prepare the inoculum. The optical density of the bacterial suspension for each of the test cultures before inoculation of the medium was brought to 0.5 by the Macfarland standard. The content of the microbial cells was $10^{9} \mathrm{CFU} / \mathrm{cm}^{3}$. Basic solutions of antibiotics were prepared in the concentration of $1000 \mu \mathrm{g} / \mathrm{cm}^{3}$ of the tested antibiotic, from which the double dilution series in test tubes were performed. Then, the contents of each test tube were mixed with $20 \mathrm{ml}$ of meat infusion agar and poured in sterile Petri dishes. After hardening of the medium, its surface was inoculated with the studied bacterial culture and cultivated within 18-24 h at the temperature of $37^{\circ} \mathrm{C}$. Such concentration of antibiotic that caused a complete inhibition of the growth of the test culture was accepted as MIC. MIC for rifampicin relative to MRSA isolates was $4 \mu \mathrm{g} / \mathrm{ml}$, and for vancomycin $-2 \mu \mathrm{g} / \mathrm{ml}$.

\section{Results}

From the samples of biomaterial from the patients with pathological processes of different localization, 335 strains of bacteria identified as $S$. aureus were selected. Most of the $S$. aureus isolates which made up 160 strains that constituted $47.8 \%$ of the total number of cultures were isolated from nasal discharge (Fig. 1).

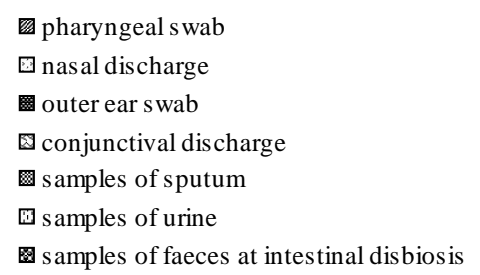


Half as many, 75 cultures (22.4\%), were isolated from the faecal samples from the patients with dysbiotic intestinal microflora disorders. 30 and 25 isolates of $S$. aureus $-9.0 \%$ and $7.5 \%$ of the total number of the isolated cultures, respectively - were the causes of diseases of the outer ear and the eye conjunctiva. The smallest number of $S$. aureus strains were isolated from pharynx and from the samples of sputum and urine - frequency of their isolation did not exceed $4.5 \%$. MRSA accounted for $50.4 \%$ out of the total number of the isolated cultures of $S$. aureus. Most often, methicillin-resistant strains were isolated from nasal discharge (57.5\% of the cultures) and faeces (50.7\% of isolates). By $40.0 \%$ of MRSA strains were isolated from conjunctival discharge, pharyngeal swab, outer ear swab and sputum. The lowest number of MRSA isolates - 33.3\% - was isolated from the samples of urine (Table 1).

During studying the antibiotic-resistant isolated MRSA strains, the following results were obtained (Fig. 2). They were found to be more resistant to gentamycin or erythromycin $-59.5 \%$. A high percentage of resistance to ciprofloxacin was retained $-53.3 \%$. The isolates of methicillin-resistant staphylococci appeared to be more sensitive to tetracycline, moxifloxacin and clindamycin. Resistance of strains to these antibiotics made up $41.4 \%$ and $33.7 \%$ respectively. Rifampicin suppressed the growth of $75.8 \%$ of MRSA cultures. They appeared to be most sensitive to vancomycin, co-trimoxazole and fusidic acid. The frequency of isolation of strains that are resistant to these antibiotics did not exceed $4.1 \%$. Linezolid caused a delay in the growth of all the studied cultures.

\section{Table 1}

Isolation of MRSA strains from samples of biomaterial from patients with pathological processes of different localization

\begin{tabular}{lc}
\hline \multicolumn{1}{c}{ Samples of biomaterial } & $\begin{array}{c}\text { Isolated MRSA strains, absolute } \\
\text { number / \% of total in samples of biomaterial }\end{array}$ \\
\hline Pharyngeal swab, $\mathrm{n}=15$ & $6 / 40.0$ \\
Outer ear swab, $\mathrm{n}=30$ & $12 / 40.0$ \\
Conjunctival discharge, $\mathrm{n}=25$ & $10 / 40.0$ \\
Samples of urine, $\mathrm{n}=15$ & $5 / 33.3$ \\
Nasal discharge, $\mathrm{n}=160$ & $92 / 57.5$ \\
Samples of sputum, $\mathrm{n}=15$ & $6 / 40.0$ \\
Samples of faeces, $\mathrm{n}=75$ & $38 / 50.7$ \\
Total, $\mathrm{n}=335$ & $169 / 50.4$ \\
\hline
\end{tabular}

It should be noted that the isolated MRSA strains differed considerably by the resistance level depending on the kind of biomaterial. MRSA strains isolated from nasal discharge appeared to be most resistant to gentamycin (68.5\% of the cultures), erythromycin (58.7\%), tetracycline (48.9\%) and ciprofloxacin (43.5\%) (Fig. 3). In the cultures from this type of biomaterial, the highest percentage of resistance to fusidic acid (3.3\%) and co-trimoxazole (7.6\%) was observed compared to the isolates isolated from clinical samples of other biotopes (Fig. 3-5). MRSA isolates were characterized by a rather high level of resistance to vancomycin and rifampicin. The number of strains that were resistant to these antibiotics was 29.3 and $5.4 \%$, respectively.

MRSA strains, isolated from the outer ear proved to be most sensitive (100.0\% of the cultures) to fusidic acid and co-trimoxazole, highly sensitive - to clindamycin and tetracycline. The percentage of the isolates resistant to the two latter antibiotics did not exceed 33.3\%. By $41.7 \%$ of methicillin-resistant strains of $S$. aureus showed resistance to moxifloxacin and erythromycin. They appeared most resistant to gentamycin and ciprofloxacin. These antibiotics suppressed the growth of MRSA isolated from the outer ear just in 41.7 and $25.0 \%$ of the cases, respectively. $16.7 \%$ of isolates were resistant even to rifampicin.

Erythromycin, which suppressed the growth of cultures in $16.7 \%$ of cases, appeared ineffective relative to the strains of MRSA isolated from the pharynx. By $33.3 \%$ of the isolates showed resistance to ciprofloxacin and clindamycin and by $16.7 \%$ - to moxifloxacin, gentamycin, tetracycline and rifampicin. Fusidic acid, co-trimoxazole and vancomycin were recognized as the most effective preparations since they led to delays of the growth of all MRSA strains isolated from the pharynx.

From the samples of faeces from the patients with dysbiotic intestinal microflora disorders, the highest percentage (71.0\%) of resistant MRSA isolates was recorded for ciprofloxacin (Fig. 4). Erythromycin and gentamycin suppressed the growth of methicillin-resistant staphylococci in $57.9 \%$ and $55.3 \%$ of cases, respectively. $47.3 \%$ of the cultures were resistant to moxifloxacin, by $36.8 \%$ - to tetracycline and clindamycin. A rather high percentage of resistant strains, isolated from faeces samples, as in previous research, was retained for the preparations of choice: rifampicin and vancomycin. $23.7 \%$ of MRSA isolates have shown resistance to rifampicin and 5.3\% - to vancomycin. MRSA strains isolated from the samples of urine were found not less resistant (Fig. 4). These cultures were found to be most resistant to ciprofloxacin (100.0\% of isolates), and erythromycin (80.0\%). Gentamycin and moxifloxacin suppressed the growth of strains in $60.0 \%$ of cases, clindamycin - in $40.0 \%$ of cases and tetracycline - in $20.0 \%$ of cases. All isolated cultures were sensitive to fusidic acid, co-trimoxazole, vancomycin and rifampicin.

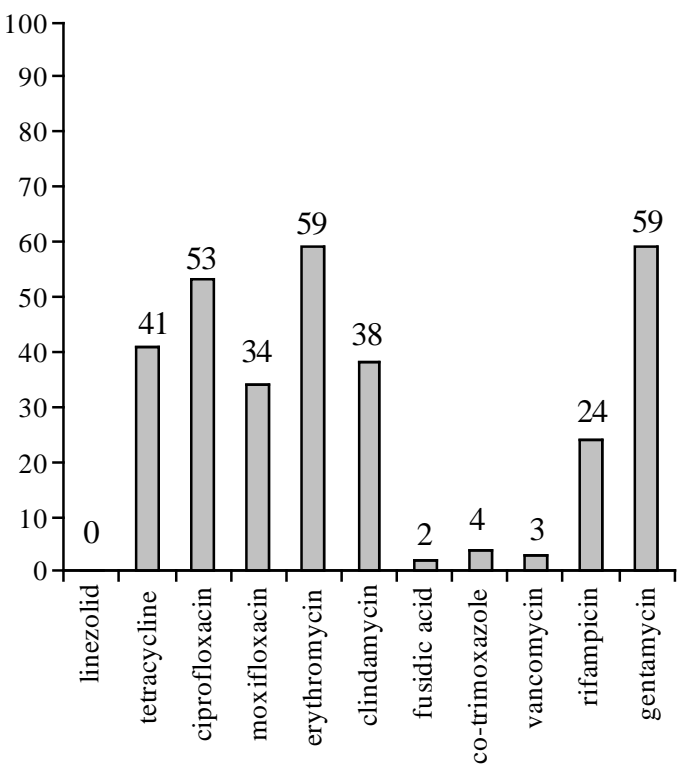

Fig. 2. Resistance to antibiotics of MRSA strains (\%), isolated from samples of biomaterial from patients with pathological processes of different localization $(n=169)$

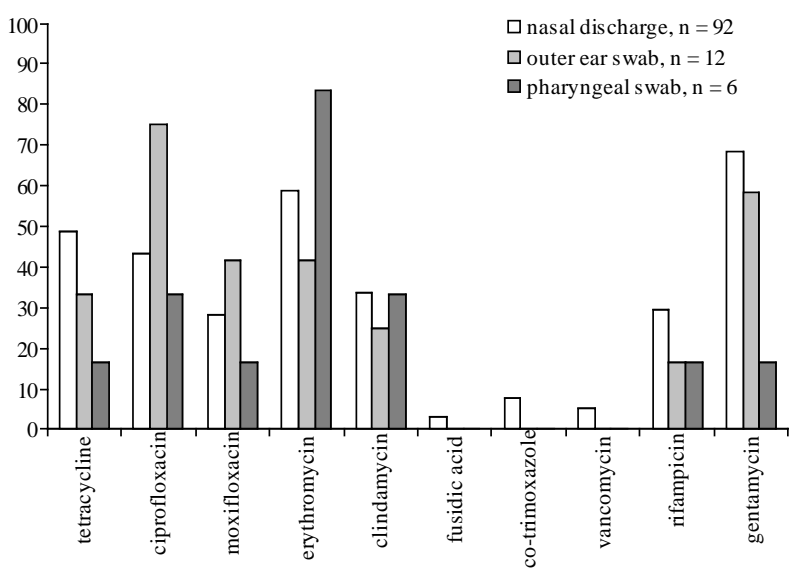

Fig. 3. Resistance to antibiotics of MRSA strains (\%), isolated from samples of nasal discharge, outer ear and pharyngeal swabs

The following results were obtained for the MRSA from sputum (Fig. 5). All the isolates isolated strains resistance to erythromycin. The degree of resistance of the strains of methicillin-resistant staphylococci to tetracycline, ciprofloxacin and clindamycin did not exceed 33.3\%, and to moxifloxacin, gentamycin and rifampicin $-16.7 \%$.

The cultures isolated from the conjunctival discharge appeared to be the most sensitive from all MRSA isolates from the patients with pathological processes of different localization (Fig. 5). The total percentage of strains resistant to antibiotics of different pharmacological groups did not exceed $50.0 \%$. Half of the cultures demonstrated resistance only to ciprofloxacin. By $40.0 \%$ of resistant isolates were obser- 
ved in relation to erythromycin and gentamycin, respectively, by $30.0 \%$ - to tetracycline and moxifloxacin. Even lower percentage of insensitive strains (10.0\%) was noted for rifampicin. Fusidic acid, cotrimoxazole and vancomycin proved to be most effective antibiotics for MRSA strains, isolated from sputum and conjunctival discharge.

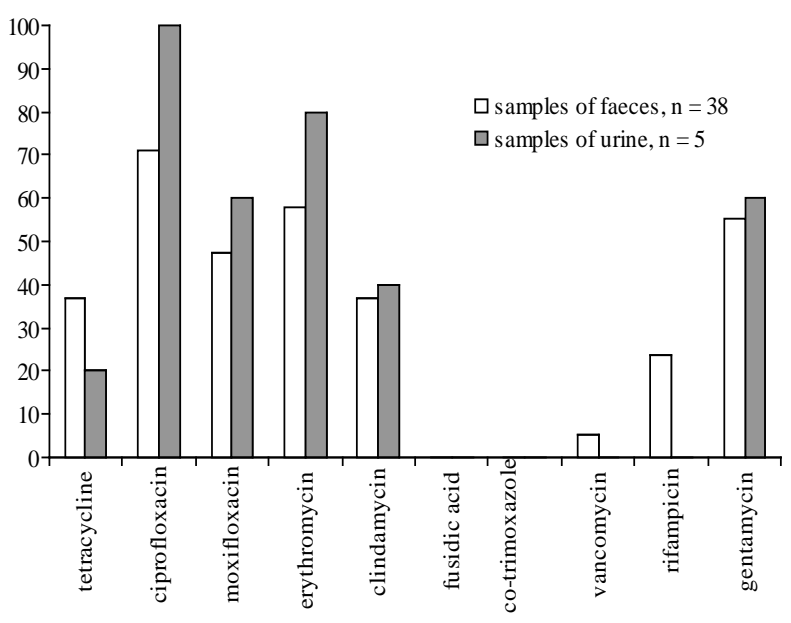

Fig. 4. Resistance to antibiotics of MRSA strains (\%), isolated from the samples of urine and faeces

Analysis of frequency of isolation of resistant variants of MRSA from different clinical samples showed that the greatest number of the antibiotic resistant cultures were the cultures isolated from the nasal cavity, faeces and urine samples. Methicillin-resistant staphylococci were isolated to a somewhat lesser extent from the samples of sputum, external ear and pharynx swabs and conjunctival discharge.

It is known that rifampicin and vancomycin are the preparations of choice for the treatment of infections caused by strains of methicillin-resistant $S$. aureus with multiple resistance to antibiotics. Our findings show that a certain number of MRSA strains isolated from the nasal cavity and faeces appeared resistant to these antibiotics. Therefore, at the next stage of our work, we determined the minimum inhibitory concentration (MIC) of rifampicin and vancomycin in relations to MRSA strains that are resistant to them (Tables 2, 3).

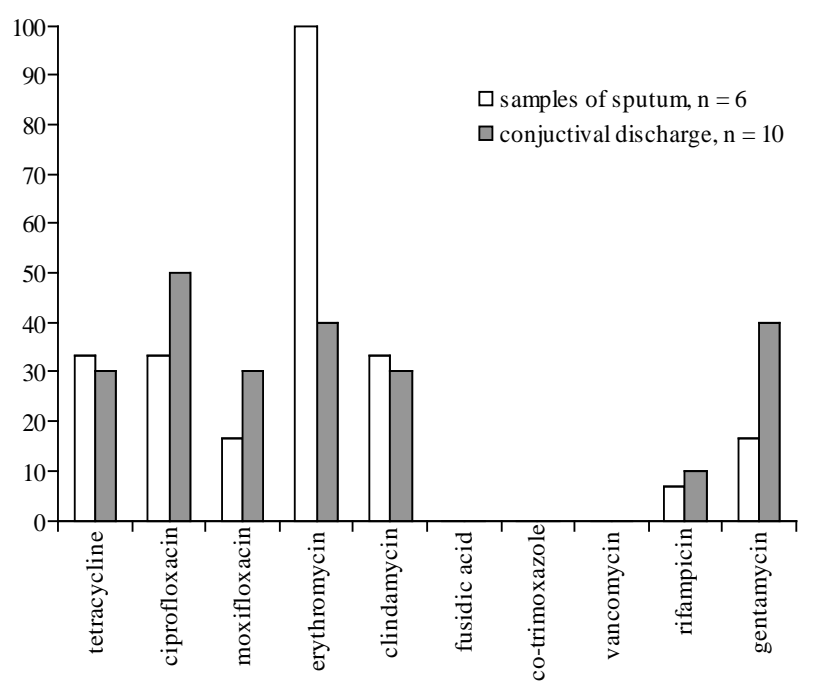

Fig. 5. Resistance to antibiotics of MRSA strains (\%), isolated from the samples of sputum and conjunctival discharge

From 27 MRSA strains, isolated from nasal discharge, the value of the minimum inhibitory concentration (MIC) of rifampicin coincided with the threshold value for the given antibiotic $(4 \mu \mathrm{g} / \mathrm{ml})$ for 18 of them (66.7\% of cultures), it exceeded the threshold value by two times $(8 \mu \mathrm{g} / \mathrm{ml})$ for 5 isolates $(18.5 \%)$, for three $(11.1 \%)$ - by four times $(16 \mu \mathrm{g} / \mathrm{ml})$, and for one (3.7\%) - by 8 times (32 $\mu \mathrm{g} / \mathrm{ml})$ (Table 2$)$. From 9 MRSA strains isolated from the faeces at intestinal dysbioses, the value of MIC of rifampicin coincided with the threshold value and made up $4 \mu \mathrm{g} / \mathrm{ml}$ for 5 cultures (55.6\%), it exceeded the threshold value by two times ( $8 \mu \mathrm{g} / \mathrm{ml})$ for 4 strains $(45.4 \%)$.

Table 2

Isolation of rifampicin-resistant MRSA strains

(absolute number / \%) with regard to MIC of rifampicin

\begin{tabular}{lcccc}
\hline \multirow{2}{*}{ Samples of biomaterial } & 4 & 8 & 16 & 32 \\
\cline { 2 - 5 } & $18 / 66.7$ & $5 / 18.5$ & $3 / 11.1$ & $1 / 3.7$ \\
\hline Nasal discharge, $\mathrm{n}=27$ & $5 / 55.6$ & $4 / 45.4$ & $0 / 0$ & $0 / 0$ \\
Samples of faeces, $\mathrm{n}=9$ & & &
\end{tabular}

Table 3

Isolation of vancomycin-resistant MRSA strains

(absolute number / \%) with regard to MIC of vancomycin

\begin{tabular}{lcccc}
\hline \multirow{2}{*}{ Samples of biomaterial } & \multicolumn{4}{c}{ MIC, $\mu \mathrm{g} / \mathrm{ml}$} \\
\cline { 2 - 5 } & $3 / 60.0$ & $1 / 20.0$ & $1 / 20.0$ & $0 / 0$ \\
\hline Nasal discharge, $\mathrm{n}=5$ & $2 / 100.0$ & $0 / 0$ & $0 / 0$ & $0 / 0$ \\
Samples of faeces, $\mathrm{n}=2$ &
\end{tabular}

From five MRSA strains isolated from the nasal discharge, the value of minimum inhibitory concentration (MIC) of vancomycin coincided with the threshold value for the given antibiotic $(2 \mu \mathrm{g} / \mathrm{ml})$ for three of them (60.0\% of cultures), for one isolate (20.0\%) it exceeded the threshold value by two times $(4 \mu \mathrm{g} / \mathrm{ml})$, for another $(20.0 \%)$ - by four times ( $8 \mu \mathrm{g} / \mathrm{ml}$ ) (Table 3). Methicillin-resistant strains of staphylococci, isolated from faeces at intestinal dysbioses also proved resistant to vancomycin. In this case, the value of MIC of vancomycin coincided with threshold value and was equal to $2 \mu \mathrm{g} / \mathrm{ml}$.

\section{Discussion}

In modern medical practice, the problem of propagation of community-associated methicillin-resistant strains of $S$. aureus, characterrized by multiple resistance to antibiotics, including the preparations of choice, is becoming increasingly relevant (Jang et al., 2009; Marimuthu et Harbarth, 2014; Lawes et al., 2015; van Kleef et al., 2017; Hamdan-Partida et al., 2018). According to the results of our studies, it was found that the isolated MRSA strains accounted for $50.4 \%$ of the total number of cultures of $S$. aureus isolated from the patients with pathological processes of different localization. The obtained data prove the results, obtained by other researchers, according to which the frequency of detection of communityassociated MRSA isolates makes up from 25.0 to $80.0 \%$ (Skov et al., 2012; Gostev et al., 2015; Lawes et al., 2015; Sirichoat et al., 2016).

Analysis of the level of antibiotic resistance of the isolated methicillin-resistant strains of $S$. aureus allowed us to divide conditionally all the tested preparations into three groups according to the degree of resistance of microbial cultures to them. The first group included the preparations with the highest level of resistance of MRSA strains to them: erythromycin, ciprofloxacin, gentamycin. The total number of MRSA isolates resistant to them was more than 50.0\% (53.3-59.2\%). The second group included the preparations with relatively high antimicrobial activity: tetracycline, clindamycin, moxifloxacin. The level of resistance of the isolated cultures to them was in the range 33.3$41.4 \%$. The highest antibacterial activity was shown by: fusidic acid and co-trimoxazole, included to the third group. More than $95.0 \%$ of the isolated MRSA strains were sensitive to fusidic acid and cotrimoxazole, which makes it possible to use these preparations in combination with other antibiotics for treatment of the infections, caused by MRSA.

The isolated MRSA strains differed significantly in terms of resistance depending on the biomaterial taken, which is possible due to specificity of the treatment of infections of different localization. The strains of methicillin-resistant staphylococci isolated from the nasal cavity, outer ear swab and samples of urine appeared to be most resistant to gentamycin; those isolated from samples of urine and sputum appeared to be most sensitive to erythromycin. The MRSA strains isolated from the outer ear swab, conjunctival discharge, samples of urine and faeces appeared to be most sensitive to ciprofloxacin. 
Rifampicin- and vancomycin-resistant strains of methicillin-resistant staphylococci were most often isolated from the clinical samples taken from the nasal cavity and faeces at intestine dysbioses. But, despite the fact that only $24.2 \%$ of the isolates were found resistant to rifampicin and $3.0 \%$ - to vancomycin, it is necessary to refer them to a separate group of preparations according to the degree of resistance of the microbial cultures to them, since rifampicin and vancomycin are the first preparations of choice for the therapy of infections caused by multiresistant isolates of methicillin-resistant staphylococci (Zhang et al., 2015; Zheng et al., 2018). On this basis, when isolating the MRSA strains that are resistant to vancomycin and rifampicin, it is necessary to determine MIC of these antibiotics in relation to the studied isolates of methicillin-resistant staphylococci.

It should be noted that a decrease in susceptibility to rifampicin is considered by some researchers as a predictor of formation of resistance to glycopeptide preparations and, above all, vancomycin, which ranks first among the drugs of choice for the therapy of the infections, caused by multiresistant MRSA strains. According to the literature, resistance to rifampicin is associated with mutations in the regulatory proB gene that encodes $\beta$-subunit of RNA-polymerase. It is believed that it is this gene that participates in formation of vancomycin-resistant variants (Howden et al., 2014; Li et al., 2016; Brinkman et al., 2017).

Since the mid 1990s, reports about the circulation of MRSA strains with decreased susceptibility to vancomycin - VISA, hVISA and VRSA - started to appear. The latter are characterized by a high level of resistance (MIC $\geq 64 \mu \mathrm{g} / \mathrm{ml}$ ) and are found rarely (only 20 isolates in the world were described) as a result of acquisition by staphylococci of resistance determinants from enterococci. VISA and hVISA are distinguished by low-levels of MIC (4-8 and 2-4 $\mu \mathrm{g} / \mathrm{ml}$, respectively), but are spread widely enough (Howden, 2005; Appelbaum, 2007).

The research, carried out by us, revealed $55.5 \%$ MRSA strains from the faeces of patients with dysbiotic intestinal microflora disorders, for which MIC of rifampicin coincided with the threshold value for this antibiotic and $44.5 \%$ - with the excess of the threshold by 2 times $(4 \mu \mathrm{g} / \mathrm{ml}) .22 .2 \%$ of them were characterized by the critical level of susceptibility to vancomycin (MIC $\geq 2 \mu \mathrm{g} / \mathrm{ml}$ ). From rifampicin- and vancomycin-resistant MRSA cultures, isolated from the nasal cavity, MIC of rifampicin coincided with the threshold value for this antibiotic for $66.7 \%$ of them and exceeded the threshold at least by 2 times for $33.3 \%$ of them. $11.1 \%$ of them were characterized by the critical level of susceptibility to vancomycin (MIC $\geq 2 \mu \mathrm{g} / \mathrm{ml}$ ) and by $3.7 \%$ of the strains exceeded MIC by 2 and 4 times, respectively ( 4 and $8 \mu \mathrm{g} / \mathrm{ml}$ ). This is consistent with the data, obtained by other researchers (Tremblay et al., 2013; Gostev et al., 2015).

Thus, the obtained results of evaluation of the level of antibiotic resistance of MRSA strains, as a whole, and to preparation of choice (rifampicin and vancomycin) with regard to their MIC, in particular, prove the necessity of the strict control over the frequency of isolation and propagation of community-associated methicillin-resistant strains of $S$. aureus with the aim of limiting their circulation among the population, development of measures to prevent an increase in resistance to antibiotics and introduction of the new therapy standards.

It should be noted that all MRSA strains isolated by us appeared sensitive to linezolid. According to the results of the monitoring studies, linezolid is characterized by a significant clinical efficiency relative to MRSA, compared to rifampicin and vancomycin. It has a wide range of actions and demonstrates high activity in relation to aerobic and anaerobic gram-positive microorganisms, including methicillin-resistant staphylococci and enterococci (MRSA and MRSE). Therefore, it can be regarded as an alternative preparation for the treatment of infections caused by multiresistant MRSA strains.

\section{Conclusions}

In our study of samples of biomaterial from the patients with pathological processes of different localization, we isolated 335 strains of bacteria identified as $S$. aureus, out of which methicillin-resistant strains composed $50.4 \%$. Most often, MRSA were isolated from nasal discharge (57.5\% of the cultures) and faeces of patients with dysbiotic intestinal microflora disorders (50.7\% of isolates). By $40.0 \%$ of MRSA strains were isolated from the conjunctival discharge, pharyngeal and outer ear swabs and sputum. Their smallest number (33.3\%) was isolated from the urine samples.

MRSA strains appeared most resistant to gentamycin, erythromycin (59.5\% of the cultures) and ciprofloxacin (53.3\% of isolates). They appeared most sensitive to vancomycin, co-trimoxazole and fusidic acid. The frequency of isolation of the cultures that are resistant to these antibiotics did not exceed $4.1 \%$. Linezolid caused a delay in the growth of all the studied isolates.

The isolated MRSA strains differed significantly by the resistance level depending on the kind of the biomaterial taken. The MRSA strains isolated from the nasal cavity, outer ear swab, samples of urine, sputum and faeces appeared to be most resistant to the tested antimicrobial preparations.

Rifampicin- and vancomycin-resistant strains of methicillinresistant staphylococci (21.3\% of the total number of MRSA) were most often isolated from the clinical samples taken from the nasal cavity and faeces at intestinal dysbioses. When determining the MIC of rifampicin and vancomycin, which are antibiotics of choice in the treatment of infections caused by multiresistant MRSA, it was found that MIC of rifampicin coincided with the threshold value for this antibiotic for $55.5 \%$ of MRSA strains from the faeces of patients with dysbiotic disturbances of the intestinal microflora and exceeded the threshold value by 2 times $(4 \mu \mathrm{g} / \mathrm{ml})$ for $44.5 \%$ of them. $22.2 \%$ of them were characterized by the critical level of susceptibility to vancomycin (MIC $\geq 2 \mu \mathrm{g} / \mathrm{ml}$ ). From rifampicin- and vancomycinresistant MRSA strains, isolated from the nasal cavity, MIC of rifampicin coincided with the threshold value for this antibiotic for $66.7 \%$ of the cultures and exceeded it at least by 2 times for $33.3 \%$ of the cultures. $11.1 \%$ of them were characterized by a critical level of susceptibility to vancomycin (MIC $\geq 2 \mu \mathrm{g} / \mathrm{ml}$ ) and by $3.7 \%$ of the strains exceeded MIC by 2 and 4 times, respectively ( 4 and $8 \mu \mathrm{g} / \mathrm{ml}$ ).

\section{References}

Aguayo-Reyes, A., Quezada-Aguiluz, M., Mella, S., Riedel, G., Opazo-Capurro, A., Bello-Toledo, H., Dominguez, M., \& González-Rocha, G. (2018). Molecular basis of methicillin-resistance in Staphylococcus aureus. Revista Chilena de Infectología, 35(1), 7-14.

Appelbaum P. (2007). Reduced glycopeptide susceptibility in methicillin-resistant Staphylococcus aureus (MRSA). International Journal of Antimicrobial Agent, 30(5), 398-408.

Bakthavatchalam, Y., Veeraraghavan, B., Devanga Ragupathi, N., Babu, P., Munuswamy, E., \& David, T. (2017). Draft genome sequence of reduced teicoplanin-susceptible and vancomycin-heteroresistant methicillin-resistant Staphylococcus aureus from sepsis cases. Journal of Global Antimicrobial Resistance, 8, 169-171.

Bakthavatchalam, Y., Veeraraghavan, B., Peter, J., Rajinikanth, J., Inbanathan, F., Devanga Ragupathi, N., \& Rajamani Sekar, S. (2016). Novel observations in 11 heteroresistant vancomycin-intermediate methicillin-resistant Staphylococcus aureus strains from South India. Genome Announcements, 4(6), e01425-16.

Becker, K., van Alen, S., Idelevich, E., Schleimer, N., Seggewiß, J., Mellmann, A., Kaspar, U., \& Peters, G. (2018). Plasmid-encoded transferable mecBmediated methicillin resistance in Staphylococcus aureus. Emerging Infectious Diseases, 24(2), 242-248.

Brinkman, C., Schmidt-Malan, S., Mandrekar, J., \& Patel, R. (2017). Rifampinbased combination therapy is active in foreign-body osteomyelitis after prior rifampin monotherapy. Antimicrobial Agents and Chemotherapy, 61(2), e01822-16.

DeLeo, F., Otto, M., Kreiswirth, B., \& Chambers, H. (2010). Community-associated meticillin-resistant Staphylococcus aureus. Lancet, 375(9725), 1557-1568.

Dhanoa, A., Singh, V. A., Mansor, A., Yusof, M. Y., Lim, K. T., \& Thong, K. L. (2012). Acute haematogenous community-acquired methicillin-resistant Staphylococcus aureus osteomyelitis in an adult: Case report and review of literature. BMC Infectious Diseases, 12, 270.

Ehelepola, N., Rajapaksha, R., Dhanapala, D., Thennekoon, T., \& Ponnamperuma, S. (2018). Concurrent methicillin-resistant Staphylococcus aureus septicemia and pyomyositis in a patient with dengue hemorrhagic fever: A case report. BMC Infectious Diseases, 18(1), 99.

García-Garrote, F., Cercenado, E., Marín, M., Bal, M., Trincado, P., Corredoira, J., Ballesteros, C., Pita, J., Alonso, P., \& Vindel, A. (2014). Methicillin- 
resistant Staphylococcus aureus carrying the mecC gene: Emergence in Spain and report of a fatal case of bacteraemia. Journal of Antimicrobial Chemotherapy, 69(1), 45-50.

Gomes, D., Ward, K., \& LaPlante, K. (2015). Clinical implications of vancomycin heteroresistant and intermediately susceptible Staphylococcus aureus. Pharmacotherapy, 35(4), 424-432.

Hamdan-Partida, A., González-García, S., de la Rosa García, E., \& BustosMartínez, J. (2018). Community-acquired methicillin-resistant Staphylococcus aureus can persist in the throat. International Journal of Medical Microbiology, in press.

Hibbitts, A., \& O'Leary, C. (2018). Emerging nanomedicine therapies to counter the rise of methicillin-resistant Staphylococcus aureus. Materials, 11(2), e321.

Howden, B. (2005). Recognition and management of infections caused by vancomycin-intermediate Staphylococcus aureus (VISA) and heterogenous VISA (hVISA). Internal Medicine Journal, 35(2), 136-140.

Howden, B., Peleg, A., \& Stinear, T. (2014). The evolution of vancomycin intermediate Staphylococcus aureus (VISA) and heterogenous-VISA. Infection, Genetics and Evolution, 21, 575-582.

Jang, H., Kim, S., Kim, K., Kim, C., Lee, S., Song, K., Jeon, J., Park, W., Kim, H., Park, S., Kim, N., Kim, E., Oh, M., \& Choe, K. (2009). Salvage treatment for persistent methicillin-resistant Staphylococcus aureus bacteremia: Efficacy of linezolid with or without carbapenem. Clinical Infectious Diseases, 49(3), 395-401.

Khan, A., Wilson, B., \& Gould, I. (2018). Current and future treatment options for community-associated MRSA infection. Expert Opinion on Pharmacotherapy, 19(5), 457-470.

Lawes, T., Lopez-Lozano, J., Nebot, C., Macartney, G., Subbarao-Sharma, R. Dare, C., Wares, K., \& Gould, I. (2015). Effects of national antibiotic stewardship and infection control strategies on hospital-associated and community-associated meticillin-resistant Staphylococcus aureus infections across a region of Scotland: A non-linear time-series study. The Lancet. Infectious Diseases, 15(12), 1438-1449.

Li, J., Feßler, A., Jiang, N., Fan, R., Wang, Y., Wu, C., Shen, J., \& Schwarz S. (2016). Molecular basis of rifampicin resistance in multiresistant porcine livestock-associated MRSA. Antimicrobial Agents and Chemotherapy, 71(11), 3313-3315.

Marimuthu, K., \& Harbarth, S. (2014). Screening for methicillin-resistant Staphylococcus aureus... all doors closed? Current Opinion in Infectious Diseases, 27(4), 356-362.

Miller, W., Bayer, A., \& Arias, C. (2016). Mechanism of action and resistance to daptomycin in Staphylococcus aureus and enterococci. Cold Spring Harbor Perspectives in Medicine, 6(11), a026997.

Oh, E., Bae, J., Kumar, A., Choi, H., \& Jeon, B. (2018). Antioxidant-based synergistic eradication of methicillin-resistant Staphylococcus aureus (MRSA) biofilms with bacitracin. International Joumal of Antimicrobial Agents, in press.

Para, R., Fomda, B., Jan, R., Shah, S., \& Koul, P. (2018). Microbial etiology in hospitalized North Indian adults with community-acquired pneumonia. Lung India: Official Organ of Indian Chest Society, 35(2), 108-115.
Pardos de la Gandara, M., Borges, V., Chung, M., Milheiriço, C., Gomes, J. P., de Lencastre, H., \& Tomasz, A. (2018). Genetic determinants of highlevel oxacillin resistance in MRSA. Antimicrobial Agents and Chemotherapy, 62(4), AAC.00206-18.

Rybak, J., Marx, K., \& Martin, C. (2014). Early experience with tedizolid: Clinical efficacy, pharmacodynamics, and resistance. Pharmacotherapy, 34(11), 1198-1208.

Sakoulas, G., Rose, W., Berti, A., Olson, J., Munguia, J., Nonejuie, P., Sakoulas, E., Rybak, M., Pogliano, J., \& Nizet, V. (2017). Classical $\beta$-lactamase inhibitors potentiate the activity of daptomycin against methicillin-resistant Staphylococcus aureus and colistin against Acinetobacter baumannii. Antimicrobial Agents and Chemotherapy, 61(2), e01745-16.

Sirichoat, A., Lulitanond, A., Kanlaya, R., Tavichakorntrakool, R., Chanawong, A., Wongthong, S., \& Thongboonkerd, V. (2016). Phenotypic characteristics and comparative proteomics of Staphylococcus aureus strains with different vancomycin-resistance levels. Diagnostic Microbiology and Infectious Disease, 86(4), 340-344.

Skov, R., Christiansen, K., Dancer, S., Daum, R., Dryden, M., Huang, Y., \& Lowy, F. (2012). Update on the prevention and control of communityacquired meticillin-resistant Staphylococcus aureus (CA-MRSA). International Journal of Antimicrobial Agents, 39(3), 193-200.

Tong, S., Davis, J., Eichenberger, E., Holland, T., \& Fowler, V. (2015). Staphylococcus aureus infections: Epidemiology, pathophysiology, clinical manifestations and management. Clinical Microbiology Reviews, 28(3), 603-661.

Tran, K., \& Rybak, M. (2018). Beta-lactams combinations with vancomycin provide synergy against VSSA, hVISA, and VISA. Antimicrobial Agents and Chemotherapy, 62(5), AAC.00157-18

Tremblay, S., Lau, T., \& Ensom, M. (2013). Addition of rifampin to vancomycin for methicillin-resistant Staphylococcus aureus infections: What is the evidence? Annals of Pharmacotherapy, 47(7-8), 1045-5104.

van Kleef, E., Luangasanatip, N., Bonten, M., \& Cooper, B. (2017). Why sensitive bacteria are resistant to hospital infection control. Wellcome Open Research, 2, 16.

Wang, J., Lai, C., Lin, H., Chen W., Shih, Y., \& Hung, C. (2013). High vancomycin minimum inhibitory concentrations with heteroresistant vancomycin-intermediate Staphylococcus aureus in meticillin-resistant S. aureus bacteraemia patients. International Journal of Antimicrobial Agents, 42(5), 390-394.

Zhang, S., Sun, X., Chang, W., Dai, Y., \& Ma, X. (2015). Systematic review and meta-analysis of the epidemiology of vancomycin-intermediate and heterogeneous vancomycin-intermediate Staphylococcus aureus isolates. PLoS One, 10(8), e0136082.

Zheng, X., Berti, A., McCrone, S., Roch, M., Rosato, A., Rose, W., \& Chen, B. (2018). Combination antibiotic exposure selectively alters the development of vancomycin intermediate resistance in Staphylococcus aureus. Antimicrobial Agents and Chemotherapy, 62(2), e02100-17. 